Mediterránea, 5 - Págs. (3 - 22), Año 1981

\title{
LA VEGETACIÓN DE LAS DUNAS LITORALES Y SU RELACIÓN CON LA MORFOLOGÍA DUNAR Y EL GRADIENTE DE INFLUENCIA MARINA.
}

\author{
Por J. García-Albá y M. Morey
}

Las dunas litorales son ecosistemas singulares caracterizados por poseer suelos brutos no evolucionados o con perfil poco diferenciado, de textura netamente arenosa y con o sin horizonte de humus diferenciado (DUCHAUFOUR, 1975). Estas características edáficas, unidas a su proximidad al mar, hacen que solo un número relativamente limitado de especies vegetales sean capaces de vivir en estas condiciones ambientales, por lo cual la flora propia de estos ecosistemas es relativamente uniforme y diferencial respecto a los ecosistemas próximos, estando constituída esencialmente por especies psammófitas, xerofitas, halofitas, oligotrofas y resistentes al viento y a la movilidad del sustrato.

Las formaciones de dunas litorales han sido consideradas tradicionalmente como lugares improductivos y faltos de interés económico; pero modernamente se ha descubierto una serie de valores de tales ecosistemas, que van desde su interés como fuente de arena para la construcción y la industria hasta su aprovechamiento como zona recreativa con capacidad de atracción turística, alcanzando los terrenos dunares precios muy elevados para urbanizaciones. Tales aprovechamientos producen efectos negativos sobre estos ecosistemas, que van desde la degradación más o menos acusada (nitrofilia inducida por la simple concentración veraniega de personas que acuden a la playa, con aparición de especies ruderales) hasta la destrucción parcial a causa de los areneros o incluso total por las urbanizaciones. Por ello hemos creído interesante estudiar estos ecosistemas en algunos aspectos florísticos y ecológicos antes de que sea demasiado tarde. En los últimos años una série de inves- 
tigadores españoles han dedicado sus esfuerzos a este problema (ALVAREZ, 1972; LORIENTE, 1974; LORIENTE Y GONZALEZ MORALES, 1974; LOSA Y ALONSO, 1974).

Desde el punto de vista fitosociológico, la vegetación de las dunas litorales se ha clasificado dentro de la División Ammophilea arenariae O. Bolós, 1968, que comprende las siguientes Clases: Cakiletea maritimae R. Tx. y Preising, 1950, correspondiente a las playas, con el Orden Euphorbietalia peplis, y Ammophiletea Br.-BI. y R. Tx., 1973, con el Orden Ammophiletalia Br.-BI. (1931) 1933, correspondiente a las dunas. Ambas clases se subdividen en categorias inferiores con sus correspondientes especies características, acompañantes, etc. (LORIENTE Y GONZALEZ MORALES, 1974).

Hemos querido realizar un estudio ecológico de varios sistemas de dunas litorales de Asturias y Galicia, que incluyen lugares relativamente bien conservados y lugares más degradados con el fin de ordenar las comunidades vegetales de los distintos sistemas a partir de muestreos cuantitativos estratificados, siguiendo un gradiente perpendicular a la línea de la costa, basado en la morfología de las dunas.

\section{MATERIAL Y METODOS}

Se han estudiado tres sistemas de dunas litorales de la provincia de Oviedo (Verdicio, Xagó y El Espartol) y uno de la de Pontevedra correspondiente a la playa de La Lanzada (FIGURA 1). La toma de muestras se realizó en los meses de mayo y junio.

Se escogió un tipo de muestreo por transecciones perpendiculares a la línea de la costa (FIGURA 1), de tal modo que se realiza por tramos siguiendo el perfil de las dunas, es decir, se trataría realmente de un muestreo estratificado siguiendo un presunto gradiente ambiental perpendicular a la línea de la costa. 

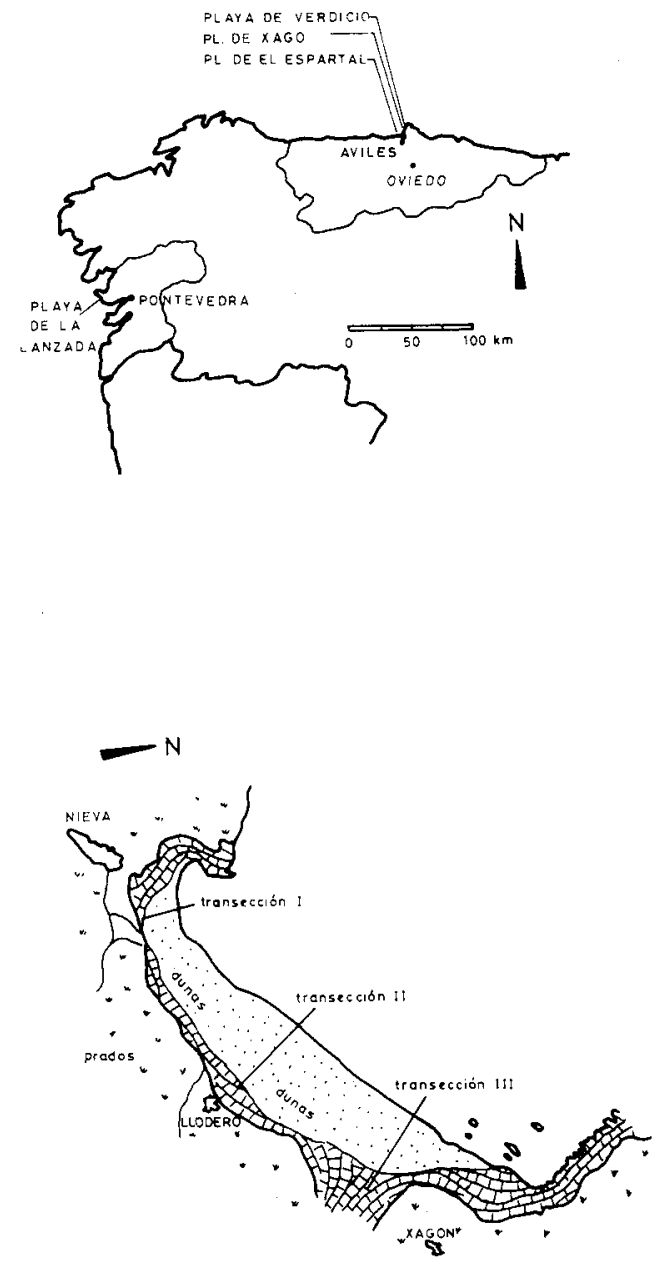

PLAYA DE XAGO


PLAYA DE VERDICIO

Figura 1.-Situación de los sistemas de dunas con indicación de las transecciones realizadas. 
En cada transición se distinguen las siguientes zonas:

A.-Playa: Comprendida entre el mar y el principio del frente de las dunas primarias. Se caracteriza por su suave pendiente, nunca superior al $15 \%$. A veces se distinguen a simple vista dos comunidades vegetales aparentemente distintas, en cuyo caso se han muestreado separadamente.

B.-Frente anterior de las dunas primarias: Comprende desde el final de la playa hasta la parte superior de las dunas primarias y se caracteriza por tener una pendiente relativamente fuerte (entre 15 y $40 \%$ en nuestro caso).

C.-Parte superior de las dunas primarias: Es la zona practicamente horizontal que forma la cima de las dunas primarias.

D.-Frente posterior de las dunas primarias: Como su nombre indica, corresponde a la zona de detrás de las dunas primarias con pendiente a veces muy acusada (hasta un $45 \%$ ).

E.-Dunas secundarias: Son las muestras más alejadas del mar y comprenden desde comunidades típicamente dunares hasta comunidades de transición a la vegetación normal de la zona (prados generalmente). El horizonte de humus ya suele estar diferenciado.

De acuerdo con esta clasificación de la morfología dunar, los lugares de muestreo pueden observarse en la FIGURA 2. 

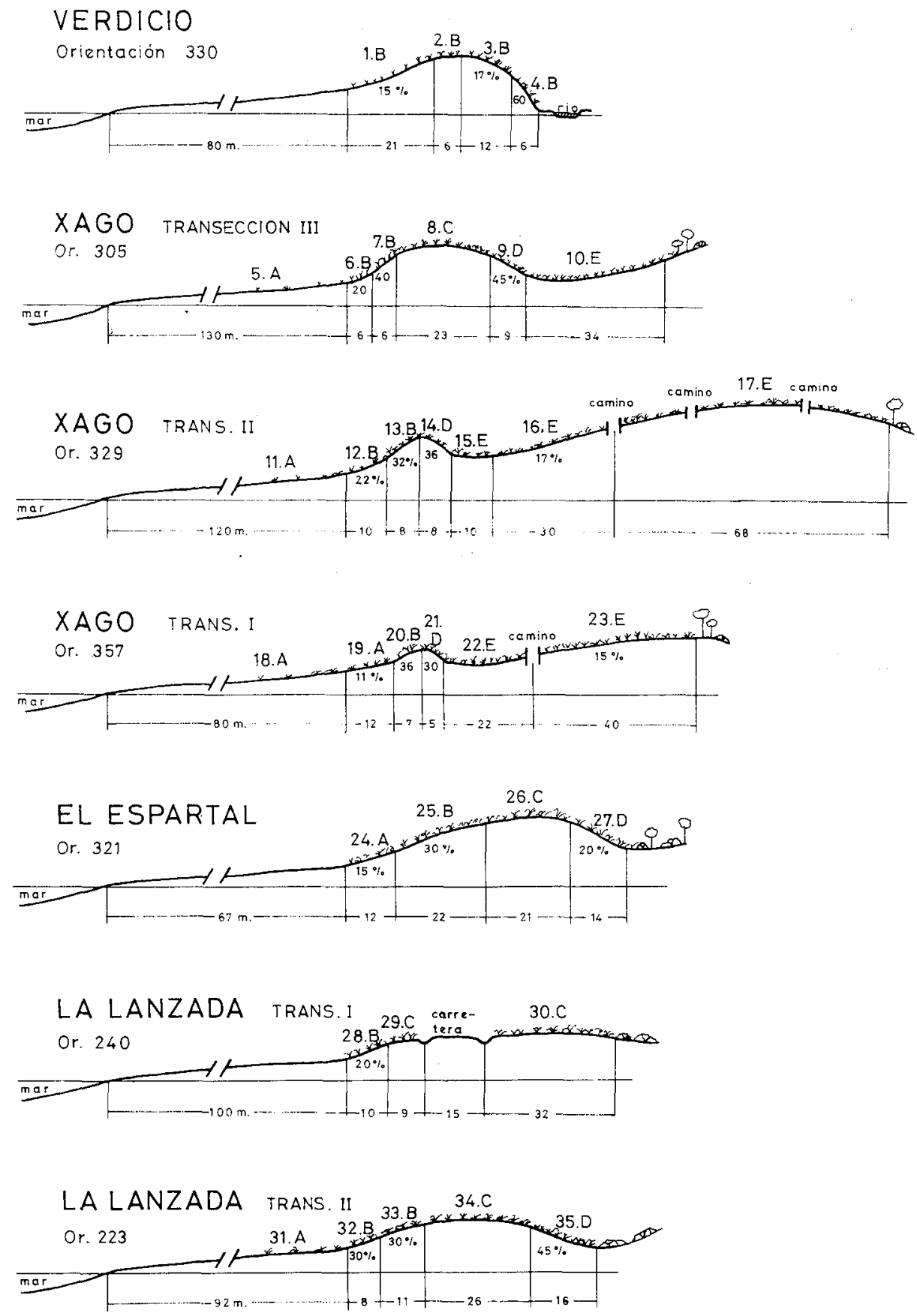

Figura 2.-Perfiles transversales de las transecciones con indicación de las zonas homogéneas (véase el texto). 
En la playa de Verdicio, la duna primaria estudiada está abierta por la parte posterior a la influencia del mar por el arroyo que discurre al fondo, de modo que sólo topográficamente puede hablarse de un frente posterior de duna primaria. Por otra parțe, la dinámica de formación de dicha duna, con un aporte continuo de arena desde el mar y un arrastre de la misma por la acción del árroyo que discurre tras ella, hacen que la arena del frente posterior se renueve periódicamente por derrumbes (G. FLOR, comunicación personal), por lo cual ninguna comunidad que allí se asiente puede progresar hacia etapas de mayor madurez. Por todo ello se han considerado todas las muestras de esta duna como pertenecientes a la zona B.

En cada lugar de muestreo se tomaron los siguientes datos: orientación, pendiente y distancia y altura sobre el nivel medio de marea. Se determino, además, la frecuencia relativa de las distintas especies usando un cuadrado de muestreo de $60 \times 60 \mathrm{cms}$ dividido en 16 subcuadrados iguales. Este cuadrado fue lánzado al azar 10 veces en cada lugar de muestreo, con lo cual las frecuencias obtenidas pueden variar entre 0 y 160 para cada especie. Para evitar el efecto de subestimación de las especies menos frecuentes, se adopto el criterio de asignar una frecuencia de uno a aquellas especies que, estando presentes, no quedaban incluídas en ninguna muestra.

En primer lugar se aplicó un análisis de componentes principales con tipificación de cocrdenadas y rotación de ejes a todas las muestras usando una computadora electrónica IBM 7070 del Centro de Cálculo Electrónico del C.S.I.C. (Madrid) y posteriormente otro análisis de componentes principales con transformación logarítmica de los datos y rotación de ejes en una computadora UNIVAC del Centro de Cálculo de la Universidad de Santiago de Compostela. En este último análisis no se incluyeron los datos de las dunas de Galicia. (SEAL, 1964; FERNANDEZ ALES y otros, 1975).

También se calculó la diversidad específica aplicando la fórmula de SHANNON (SHANNON-WEAVER, 1963).

\section{RESULTADOS Y DISCUSION}

Se identificó un total de 64 táxones, la mayor parte de ellos a nivel de especie, que se muestran con sus correspondientes frecuencias totales en la TABLA 1. Como se ve, Calystegia soldanella, Ammophila arenaria, Eryngium maritimun, Euphorbia paralias y Agropyrum junceum son las más frecuentes y al mismo tiempo las más características de estos ecosistemas (LORIENTE Y GONZALEZ MORALES, 1974). 
TABLA 1.-Relación de especies observadas, con indicación de su frecuencia relativa en tanto por ciento.

Especie

Aira caryophyllea L.

Agropyrum junceum $\mathrm{P}$. Br.

Ammophila arenaria Link

Anagallis arvensis $\mathrm{L}$.

Andriala integrifolia $\mathrm{L}$.

Anthyllis vulneraria $L$.

Arbutus unedo L.

Artemisia crithmifolia L.

Asperula cynanchica Rauhin L. var. maritima Lge.

Atriplex hastata L.

Calystegia soldanella (L.) R. Br.

Cakile maritima Scop.

Carex arenaria L.

Carpobrotus acinaciformis (L.) L. Bolus

Frecuencia

2,9

45,7

80,0

5,7

37,1

11,4

2,9

11,4

14,3

2,9

85,7

20,0

40,0

8,6

20,0

11,4

11,4

2,9

5,7

25,7

42,9

2,9

2,9

65,7

5,7

57,1

5,7

5.7

37,1

17,1

17,1 
Lagurus ovatusL.

Linaria sp.

Lotus corniculatusL.

Malcolmia littorea $\mathrm{R}$. Br.

Medicago marina $\mathrm{L}$.

14,3

Medicago suffruticosa RAM.

Musgo

14,3

Ononis reclinata $L$.

Ononis repens $\mathrm{L}$.

Ononis sp.

Otanthus maritimus (L.) Hoffgg. y Link

Phleum arenarium $\mathrm{L}$.

Pinus pinaster Ait.ssp. atlantica $\mathrm{H}$. del Villar

Plantago coronopus $\mathrm{L}$.

Poa sp.

Polygala vulgaris $L$.

Polygonum maritimum L.

14,3

Populus nigra $L$.

Pteris aquilina $L$.

14,3

Rubia peregrina L.

Salsola kali L.

Scolymus hispanicus L.

Sedum acre L.

Seseli sp.

Silene inflata $\mathrm{L}$.

Smilax aspera L.

20,0

Tamarix gallica L.

Thrincia hirta Roth.

Trifolium repens $\mathrm{L}$.

Ulex europaeus L.

Vicia sp. 
En el análisis de componentes a partir de los datos no transformados el porcentaje de información recogida por los primeros ejes es bastante aceptable, aproximándose al $50 \%$ en los tres primeros componentes.

\begin{tabular}{ccc} 
Componente & $\begin{array}{c}\text { Información } \\
\text { recogida }\end{array}$ & $\begin{array}{c}\text { Información } \\
\text { acumulada }\end{array}$ \\
\hline I & 20,00 & 20,00 \\
II & 13,97 & 33,97 \\
III & 10,16 & 44,13 \\
V & 8,29 & 52,42 \\
& 6,90 & 59,32 \\
\hline
\end{tabular}

Los factores de carga más representativos para los dos primeros componentes se muestran en la TABLA 2.

TABLA 2.-Factores de carga del primer análisis de componentes principales ordenados por sus valores absolutos decrecientes.

$\begin{array}{lll} & \text { POSITIVOS } & \text { NEGATIVOS } \\ \text { Lagurus ovatus } & \text { Agropyrum junceum } \\ & \text { Cerastium sp. } & \text { Cakile maritima } \\ & \text { Phleum arenarium } & \text { Malcolmia littorea } \\ & \text { Carex arenaria } & \text { Salsola kali } \\ \text { Asperula cynanchica } & \\ \text { Musgo } & \\ & \text { Poa sp. } & \\ & \text { Anthyllis vulneraria } & \\ & \text { Koeleria albescens } & \\ & \text { Pteris aquilina } & \\ & \text { Vulpia uniglumis } & \\ & \text { Thrincia hirta } & \\ & \text { Smilax aspera } & \\ & \text { Trifolium repens } & \\ & \text { Ononis reclinata } & \\ & \text { Andryala integrifolia } & \\ & \text { Erythraea littoralis } & \\ & \text { Chlora perfoliata } & \\ & \text { Anagallis arvensis } & \text { Agropyrum junceum } \\ & \text { Helichrysum stoechas } & \text { Cakile maritima } \\ \text { Scolymus hispanicus } & \text { Salsola kali } \\ \text { Ononis sp. } & \text { Honkenia peploides } \\ & \text { Sedum acre } & \\ \text { Seseli sp. } & \\ \text { Malcomia littorea } & \end{array}$


La proyección de los lugares de muestreo sobre el espacio formado por los dos primeros ejes de coordenadas puede observarse en la FIGURA 3. Todas las muestras propias de playa y del frente anterior de las dunas primarias (Zonas A y B) son muy similares y están caracterizadas por la presencia de Agropyrum junceum, Cakile maritima y Salsola kali (TABLA 2). Por otra parte, se observa un comportamiento diferencial de las dunas de Galicia frente a las de Asturias,

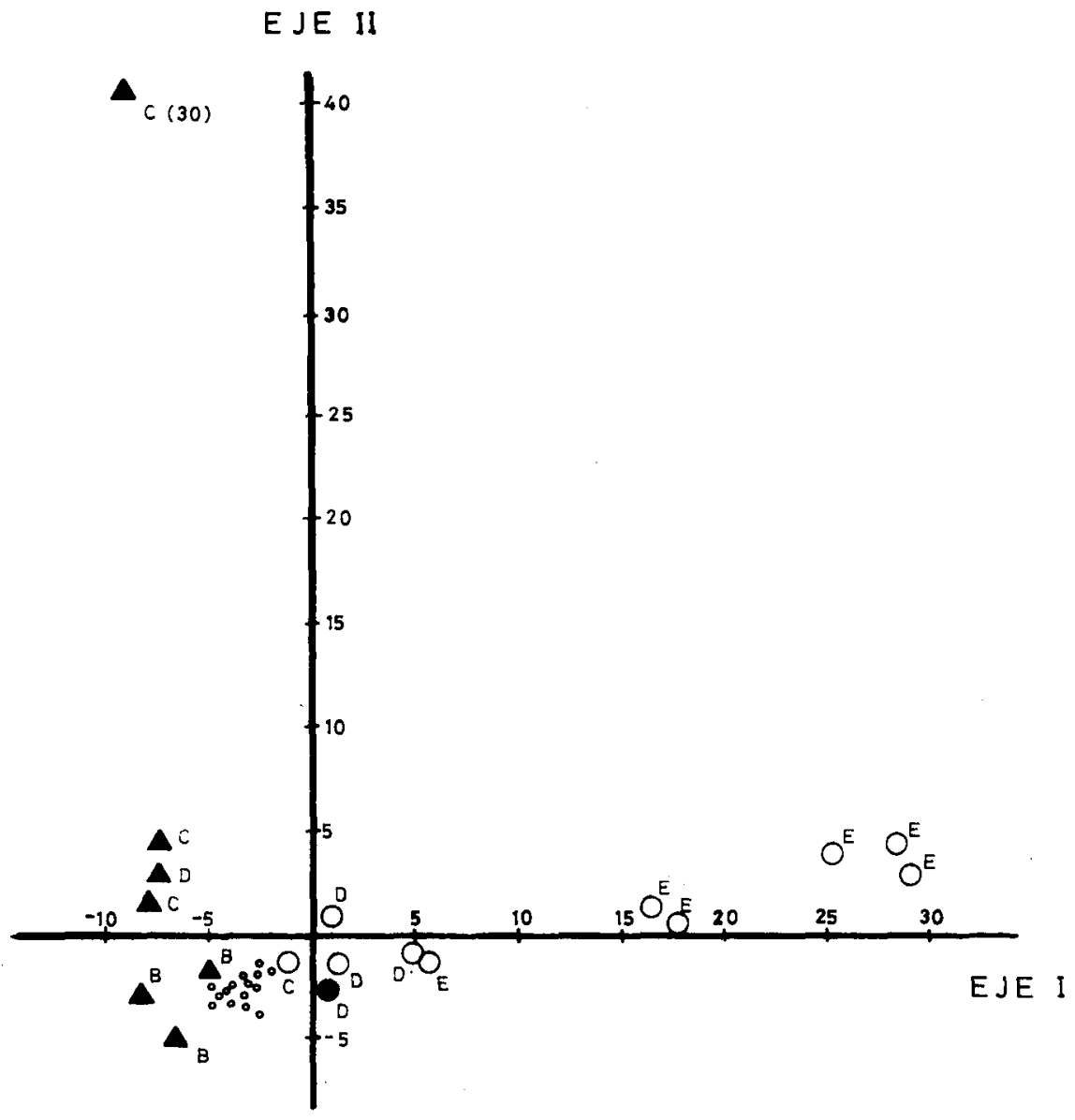

Figura 3.-Proyección de las muestras sobre los dos primeros ejes del análisis de componentes principales. Puntos: Verdicio. Círculos blancos: Xagó. Círculos negros: EI Espartal. Triángulos negros: La Lanzada. Las letras que acompañan los lugares de muestreo indican la zona del sistema dunar a que pertenecen. 
caracterizado por los valores negativos más elevados para el componente I y valores positivos también más elevados para el II; es decir, que se diferencian fundamentalmente por la presencia de Anagallis arvensis, Helichrysum stoechas, Scolymus hispanicus, Ononis sp.; Sedum acre, Seseli sp. y Malcolmia littorea. Estas especies, efectivamente, se han encontrado únicamente en las dunas de Galicia y, en conjunto, parecen de distribución más mediterránea que las demás. Cabe pensar en este caso en un efecto climático y biogeográfico, puesto que las dunas gallegas estudiadas están enclavadas en una zona de clima mediterráneo subhúmedo con tendencia a atlántico europeo (Subregión IV (V)), mientras que las dunas asturianas están en la Subregión V (VI) o de clima atlántico europeo (ALLUE ANDRADE, 1966). Realmente en el mapa del citado autor, la playa de La Lanzada aparece en la Subregión V (VI), pero los estudios que estamos realizando en el Departamento de Ecología de la Universidad de Santiago demuestran claramente que toda la zona de las Rías Bajas pertenece al clima citado anteriormente.

El componente I marca una dirección de variación florística tal, que se suceden hacia la parte positiva los lugares de muestreo correspondientes a las sucesivas zonas desde el mar hasta el interior, marcadas por las especies factores de carga positivos para este eje (véase la TABLA 2).

Se ha visto como todos los lugares de muestreo de playa y frente anterior de las dunas primarias son muy similares y se encuentran muy agrupados en la parte negativa de ambos ejes. Ampliando la escala para poder observar mejor su distribución (FIGURA 4), se observa que las comunidades de playa se agrupan en el extremo más negativo para los dos ejes, sea cual sea el sistema dunar a que pertenecen, excepto en el caso de Galicia, que ya se ha indicado que tiene un comportamiento distinto. La muestra número 30 , correspondiente a la zona $\mathrm{C}$ de La Lanzada, queda totalmente discriminada de los demás, como si fuera florísticamente muy distinta. Cabe señalar que dicha muestra está separada de las demás de su sistema dunar por una carretera muy frecuentada, por lo cual es posible que sus diferencias floristicas sean debidas, no sólo a la posible ruderalidad impuesta por la proximidad de dicha carretera, sino también a la ruptura de la dinámica del sistema causada por dicha carretera. 


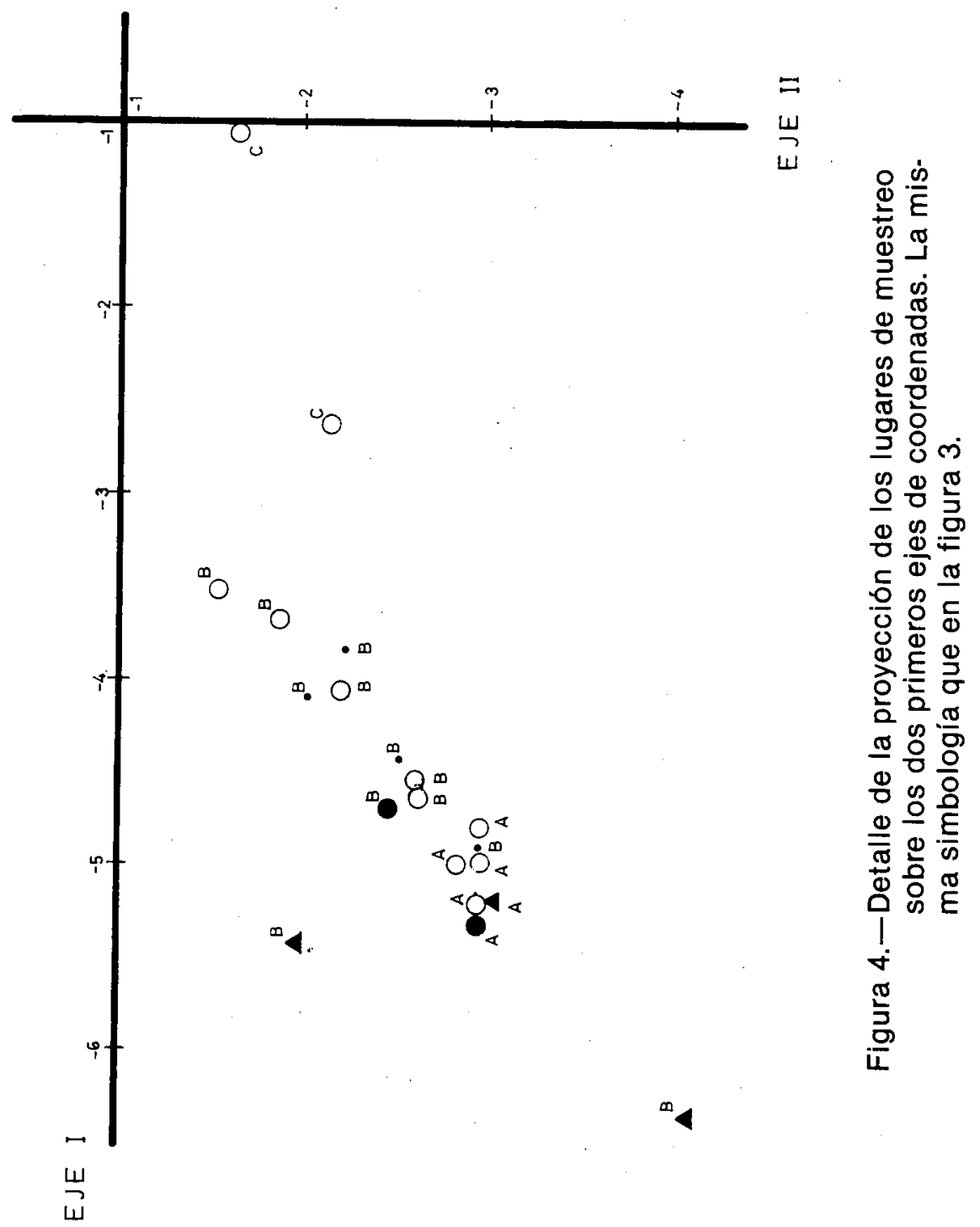


Con objeto de eliminar el posible efecto biológico provocado por las dunas gallegas, se sometieron a un nuevo análisis de componentes principales previa transformación logarítmica los datos correspondientes a las dunas asturianas. Con ello se gana información, pues el porcentaje de varianza recogido por los cinco prịmeros ejes alcanza en este nuevo análisis más del $72 \%$ frente al $59 \%$ del análisis anterior.

Los factores de carga para los dos primeros ejes de coordenadas pueden verse en la TABLA 3 y la proyección de los lugares del muestreo sobre los dos primeros ejes en la FIGURA 5.

TABLA 3.-Factores de carga del segundo análisis de componentes principales (sólo Asturias) ordenadas por sus valores absolutos decrecientes.

\section{EJE I}

EJE II

\section{POSITIVOS}

Musgo

Cerastium sp.

Anthyllis vulneraria

Ononis reclinata

Smilax aspera

Poa sp.

Aira caryophyllea

Lotus corniculatus

Pteris aquilina

Phleum arenarium

Lagurus ovatus

Trifolium repens

Asperula cynanchica

Koeleria albescens

Andriala integrifolia

Calystegia soldanella

Ammophila arenaria

Euphorbia paralias

Agropyrum iunceum

Eryngium maritimum

Carex arenaria

Vulpia uniglumis
NEGATIVOS

Calystegia soldanella

Agropyrum junceum

Ononis repens

Equisetum sp.

Crucianella maritima
Cakile maritima

Salsola kali

Polygonum maritimum 


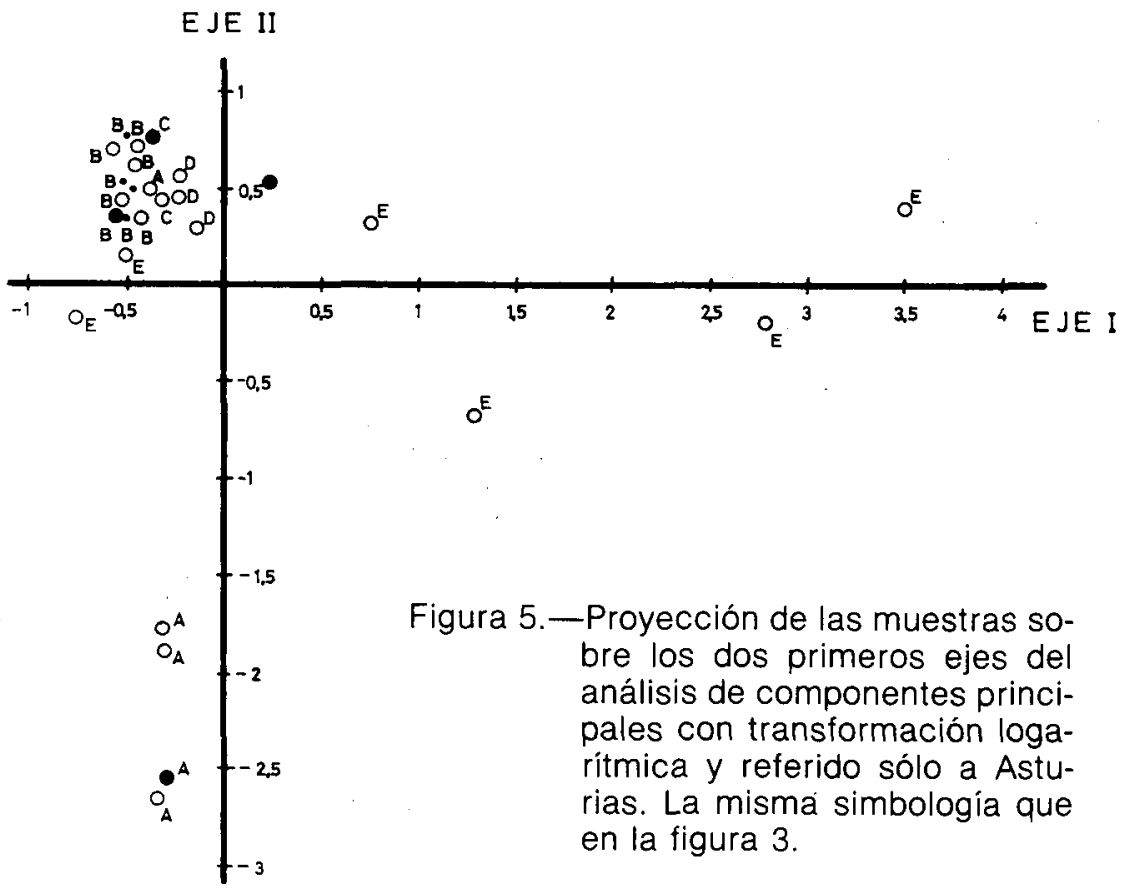

Nuevamente se observa la similitud entre todas las muestras de cada una de las zonas topográficas en que se dividieron las dunas. En este caso, las muestras de la zona de playa (A) quedan muy diferenciadas de las de la zona $B$ o del frente anterior de las dunas primarias, y están caracterizadas por la presencia de Cakile maritima, Salsola kali y Polygonum maritimum. Hay que señalar el irregular comportamiento de la muestra 19 de Xagó, que se comporta como una muestra de la zona B por la elevada frecuencia de Agropyrum junceum y Ammophila arenaria, propias de este nivel. Las muestras del frente anterior de las dunas primarias (zona B) se caracterizan por la presencia de Calystegia soldanella, Agropyrum junceum, Ammophila arenaria, Euphorbia paralias y Eryngium maritimum, fundamentalmente, mientras que las de las zonas $C$ y $D$ son relativamente parecidas a las de la zona $B$. Finalmente, las comunidades de dunas secundarias (zona E) están caracterizadas por la presencia de musgo, Cerastium sp., Anthylis vulneraria, Ononis reclinata, Smilax aspera, Poa sp., Aira cariophyllea, Lotus corniculatus, que parecen marcar una transición a comunidades pratenses.

Comparando los resultados obtenidos en los dos análisis efectuados, se observa que coinciden en lo fundamental; pero que, según sea el tipo de análisis aplicado, algunos hechos se nos presen- 
tan mejor o peor diferenciados. Así, es evidente que sólo el último de los análisis nos diferencia claramente y caracteriza bien las muestras de playa (A) y las del frente anterior de las dunas primarias (B), dejando, en cambio muy desdibujadas las diferencias entre las muestras de esta última zona y las de las zonas $\mathrm{C}$ y $\mathrm{D}$. Ello se debe a que, al realizar en el segundo análisis una transformación logarítmica de los datos, queda proporcionalmente aumentada la frecuencia, muy baja en la realidad, de las especies propias de las zonas A y $B$, con lo cual adquieren más peso estadístico en el tratamiento analítico. En cambio en el primer análisis, las muestras correspondientes a las zonas del frente posterior de las dunas primarias (D) y las de las dunas secundarias (E) quedan proporcionalmente más diferenciadas de las demás que en el último análisis.

Considerando conjuntamente todos los resultados obtenidos, pueden caracterizarse las comunidades correspondientes a las distintas zonas topográficas de las dunas estudiadas. La zona de playa (A) se caracteriza por las especies Cakile maritima, Salsola kali y Polygonum maritimum. Las comunidades del frente anterior de las dunas primarias $(B)$ se diferencian de las anteriores por la presencia de Calystegia soldanella y Agropyrum junceum y comienza a encontrarse a veces Ammophila arenaria, que, junto con Euphorbia paralias y Eryngium maritimum, caracteriza las zonas $\mathrm{C}$ y $\mathrm{D}$. Finalmente, las especies caracteristicas de las comunidades de dunas secundarias (E) dependen en gran parte de las características floristicas de la región en la que se asientan las dunas. En nuestro caso pueden asignarse como más caracteristicas el musgo, cuya presencia está ligada a la aparición de un horizonte de humus más o menos definido, Cerastium sp., Anthyllis vulneraria, Lagurus ovatus, Phleum arenarium, Ononis reclinata, Smilax aspera y Carex arenaria, entre otras. Mientras que las espécies características de la playa y frente anterior de las dunas son las mismas que señalan los fitosociólogos, las de las otras zonas corresponden, en parte, a ciertas facies localizadas en términos generales en dunas secundarias por algunos autores (LORIENTE y GONZALEZ MORALES, 1974).

Los valores obtenidos de diversidad específica oscilan entre 0,92 y 3,78 . En lineas generales, se obtienen valores más elevados en las zonas más alejadas del mar, es decir en las dunas secundarias con horizonte de humus. (FIGURA 6). Esto parece reflejar la existencia de una sucesión en sentido mar-tierra. También se observa que en la mayor parte de las transecciones aparece un descenso de la diversidad en la zona $B$, lo cual se explica si se tiene en cuenta la clara dominancia de Agropyrum junceum sobre las demás especies. Es notable la semejanza entre el perfil de diversidad de las dunas de El Espartal y La Lanzada, que son bastante semejantes entre si en cuanto a perfil topográfico e intensidad de la influencia humana. 


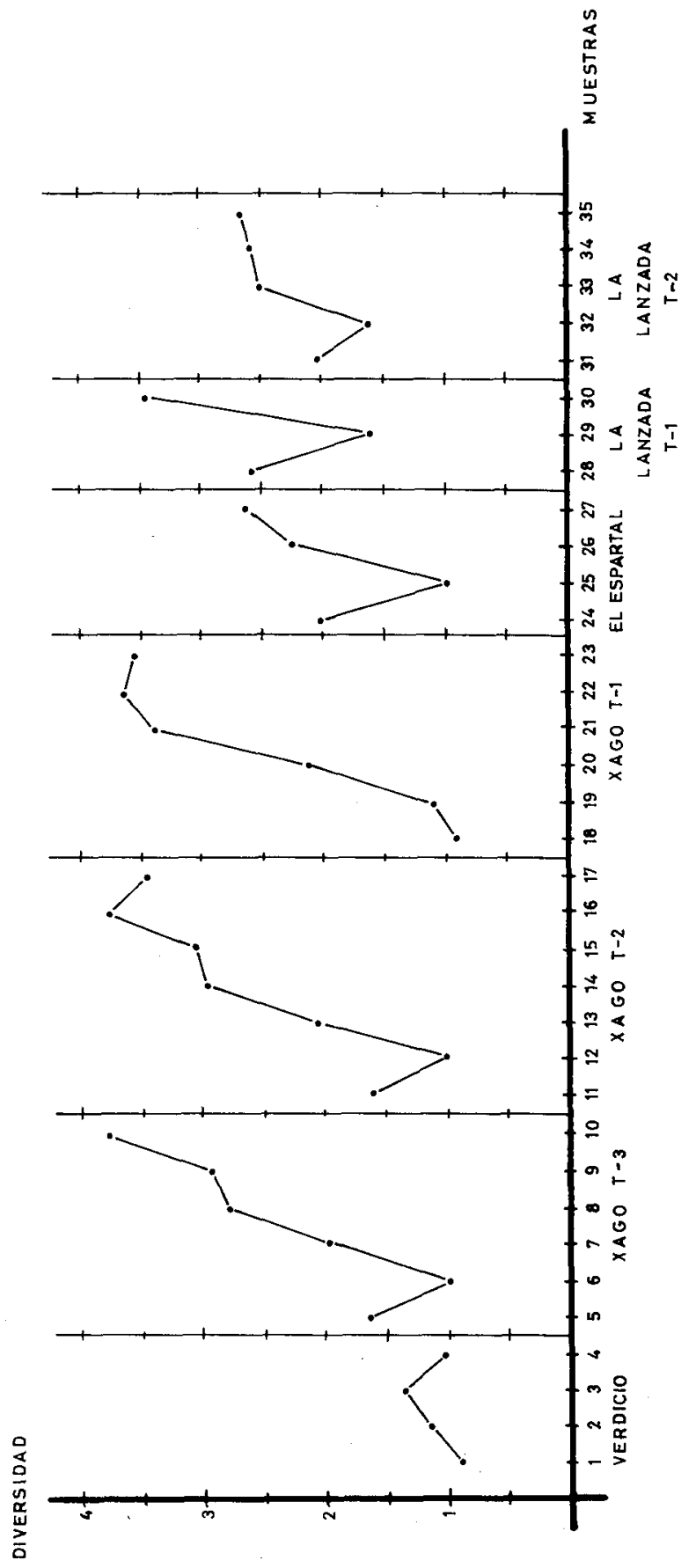

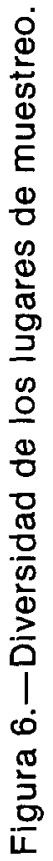


El comportamiento diferencial de las dunas de Galicia con respecto a las de Asturias, que ya quedaba bien manifiesto a través del primer análisis de componente principales, vuelve a presentarse cuando se observa el comportamiento de la diversidad frente a las coordenadas del primer componente (FIGURA 7). También en este caso, la muestra de La Lanzada $n .{ }^{\circ} 30$ se separa significativamente de todas las demás.

Agrupando los valores de diversidad de todos los lugares de muestreo semejantes, se calcularon las medias, desviaciones típicas y coeficientes de variación, que figuran en la TABLA 4. Cabe destacar el ya comentado aumento de la diversidad a medida que los lugares de muestreo están más alejados del mar y la progresiva disminución del coeficiente de variación en la misma dirección, lo cual nos indica una creciente homogeneización de las comunidades dunares en el sentido indicado, poniendo de manifiesto la existencia de una sucesión en el sentido mar-tierra. El elevado valor del coeficiente de variación de la zona $B$ puede interpretarse en función de la inestabilidad de las comunidades de dicha zona en la que Agropyrum junceum intenta continuamente fijar la duna, consiguiendo, a veces, ocupar el frente dunar completa o parcialmente, por lo cual las comunidades de esta zona oscilan a lo largo del tiempo en cuanto a grado de madurez.

TABLA 4.- Valores medios y medidas de dispersión de la diversidad específica de los distintos lugares de muestreo.

\section{Lugares de muestreo}

Playa

Frente ant. de las d. primarias

Parte sup. de las d. primarias

Frente post. de las d. prims.

Dunas secundarias

\section{Diversidad}

$$
\text { media }(\bar{H})
$$

A

B

1,56

1,52

2,55

2,88

3,53 $\mathrm{s}$

0,37

0,62

0,59

0,24

0,26
C.V. $24,0 \%$ $40,5 \%$ $23,0 \%$ $8,3 \%$ $7,4 \%$ 


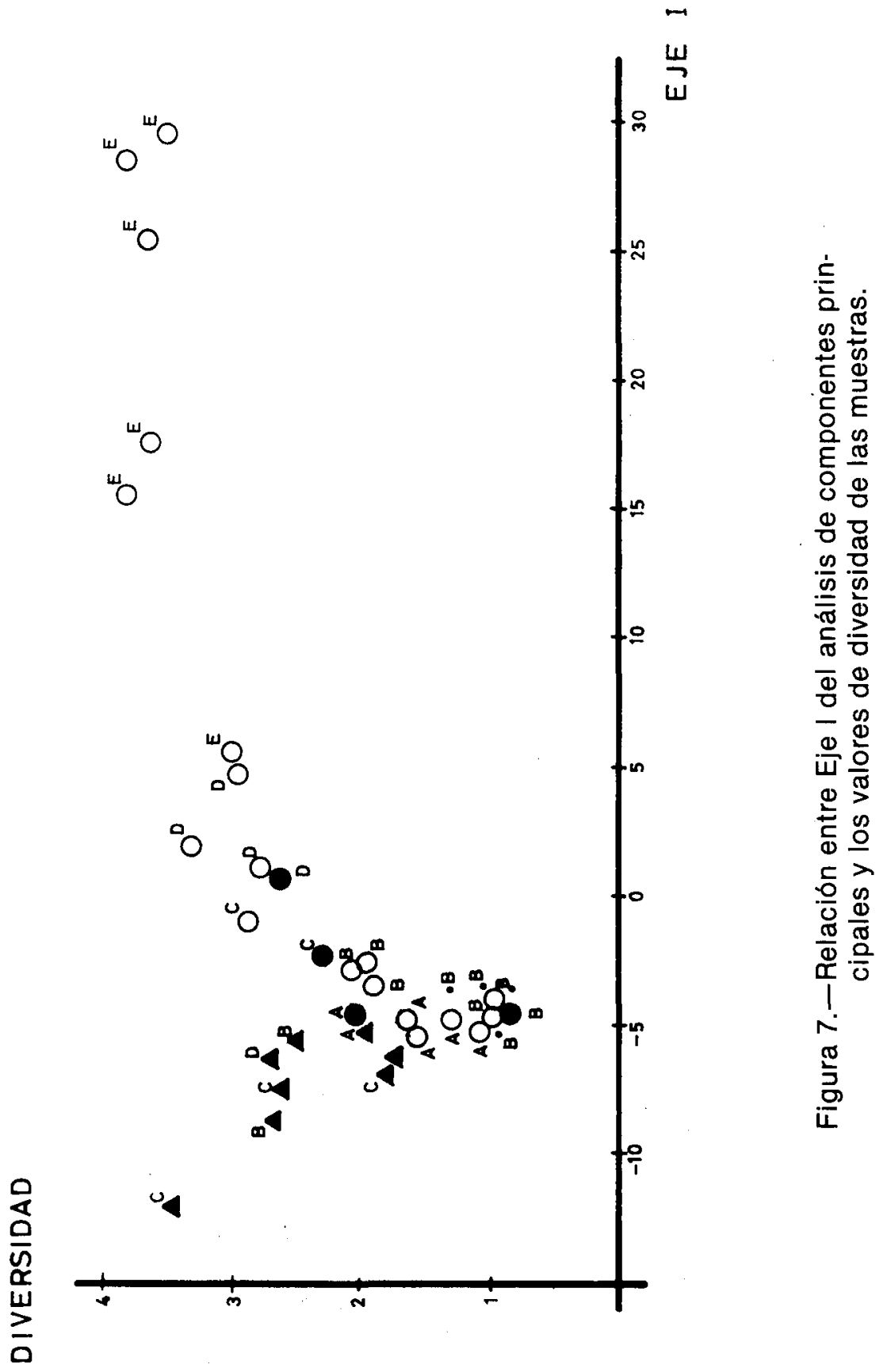




\section{RESUMEN}

Se han estudiado cuatro sistemas de dunas litorales de Asturias y Galicia por medio de un muestreo estratificado con análisis florístico cuantitativo. Los datos se sometieron a un análisis de componentes principales con y sin transformación logarítmica. Estos análisis ponen de manifiesto la existencia de una sucesión de comunidades a lo largo de un gradiente mar-tierra, siendo la propia morfología de la duna la que condiciona la distribución de dichas comunidades. Se dan las especies características de cada comunidad. Se constata un aumento de la diversidad en el sentido martierra, lo que parece reflejar un aumento de la madurez en la sucesión.

\section{SUMMARY}

THE VEGETATION OF SAND DUNES AND ITS RELATIONSHIP WITH THE DUNE MORPHOLOGY AND MARINE INFLUENCE.

The vegetation of our sand dunes in Asturias and Galicia (Spain) has been studied, sampling along gradients of marine influence from the sea inland.

A factorial analysis of principal components has been applied to all the floristical data obtained. In order to eliminate the biogeographical component, it has been also applied to the asturian data only. A succesion of plant communities from the beach inland is shown. The dune morphology itself explains the distribution of the communities. The characteristic species of each community are listed. Species diversity increases along the gradient sea-inland, reflecting the growth maturity.

Departamento de Ecología Facultad de Ciencias Universidad de Oviedo

Departamento de Ecología Facultad de Biología Universidad de Santiago 


\section{BIBLIOGRAFIA}

ALVAREZ, R. (1972). Estudio de la flora y vegetación de las playas de Galicia.

Trabajos Compostelanos de Biologia, 2: 35-66.

ALLUE ANDRADE, J.L. (1966). Subregiones Fitoclimáticas de Espaก̃a.

Ministerio de Agricultura. MADRID.

DUCHAUFOUR, P. (1975). Manual de Edafologia.

Toray-Masson. BARCELONA.

FERNANDEZ ALES, R.; SANCHO, A. Y TORRES, A. (1975). Introducción al análisis multivariante.

Depto. de Ecologia. Universidad de Sevilla.

LORIENTE, E. (1974). Vegetación y flora de las playas y dunas de la provincia de Santander.

Institución Cultural de Cantabria (C.S.I.C.) Santander.

LORIENTE, E. y GONZALEZ MORALES, J.A. (1974). Liencres: el paisaje dunar más importante del Cantábrico.

Publ. Inst. Etnogr. y Flok., 6: 213-236.

LOSA, J.M. y ALONSO, J.L. (1974). Playas arenícolas de la playa del Testal (Noya, España).

Trabajos Compostelanos de Biologia, 4: 9-23.

SEAL, H. (1964). Multivariate Statistical Analysis for Biologists. Methuen. LONDRES.

SHANNON, C.E. y WEAVER, W. (1963). The mathematical theory of communication.

University of Illinois Press. Urbana. 